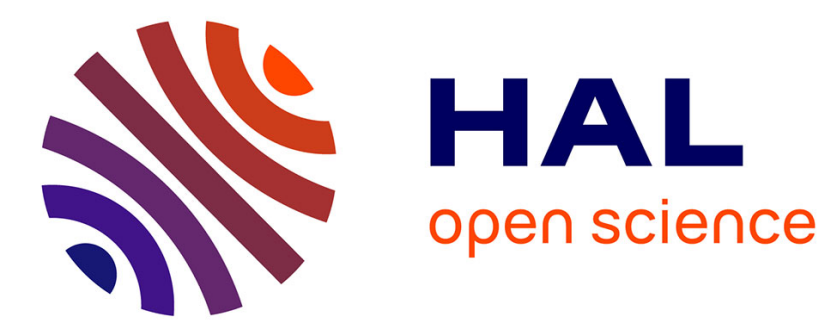

\title{
Robotic Assistance to Flexible Endoscopy by Physiological-Motion Tracking
}

Laurent Ott, Florent Nageotte, Philippe Zanne, Michel de Mathelin

\section{To cite this version:}

Laurent Ott, Florent Nageotte, Philippe Zanne, Michel de Mathelin. Robotic Assistance to Flexible Endoscopy by Physiological-Motion Tracking. IEEE Transactions on Robotics, 2011, 27 (2), pp.346359. 10.1109/TRO.2010.2098623 . hal-02377588

\section{HAL Id: hal-02377588 \\ https://hal.science/hal-02377588}

Submitted on 23 Nov 2019

HAL is a multi-disciplinary open access archive for the deposit and dissemination of scientific research documents, whether they are published or not. The documents may come from teaching and research institutions in France or abroad, or from public or private research centers.
L'archive ouverte pluridisciplinaire HAL, est destinée au dépôt et à la diffusion de documents scientifiques de niveau recherche, publiés ou non, émanant des établissements d'enseignement et de recherche français ou étrangers, des laboratoires publics ou privés. 


\title{
Robotic Assistance to Flexible Endoscopy by Physiological Motion Rejection
}

\author{
Laurent Ott, Student Member, IEEE, Florent Nageotte, Philippe Zanne, and Michel de Mathelin, Senior \\ Member, IEEE
}

\begin{abstract}
Flexible endoscopes are used in many surgical procedures and diagnostic exams, like in gastroscopy or colonoscopy. They have also been used recently for endoscopic surgical procedures using natural orifices (NOTES). Flexible endoscopes allow to access operating areas which are not easily reachable, with one small or no incision. However, their manipulation is complex especially for surgical interventions. In order to help the physicians during interventions, we propose to motorize the flexible endoscope and to partially robotize its movements. This paper explains how the endoscope can be actively stabilized with respect to an area of interest despite physiological motions by using visual servoing and repetitive control. In vivo experiments show the validity of the proposed solution for improving the manipulation of the endoscope.
\end{abstract}

Index Terms-

\section{INTRODUCTION}

$\mathbf{F}$ Lexible endoscopes are medical instruments widely used in diagnostic exams but also for endoscopic surgical procedures. They are the main instruments in gastroscopy and coloscopy where they allow to reach internal targets through natural orifices without scars. Flexible endoscopes have allowed to overcome many medical problems such as cleaning, asepsys and good visual feedback. These capabilities have been used recently for developing new endoscopic surgical techniques called NOTES (Natural Orifice Transluminal Endoscopic Surgery) [10]. In this technique, the peritoneal cavity is accessed by using a flexible endoscope which passes through natural orifices and canals (the mouth and the oesophagus, the anus and the colon, etc.) and an opening in an inner wall (the stomach, the intestine wall, etc.). This allows to accomplish treatments such as cholecystectomies without visible scars [14]. For instance, the first no visible scar transvaginal cholecystectomy procedure was carried out in 2007 using a conventional flexible endoscope [11].

Conventional flexible endoscopes used in gastro-enterology generally consist in three parts: A control handle with two navigation wheels, a flexible shaft usually more than one meter long with a circular cross section, and a distal bending tip about $10 \mathrm{~cm}$ long (see fig. 2 and 1). The tip of the endoscope has a total of 4 DOFs. The endoscope can be moved forward and backward and rotated from the proximal part. Moreover the tip of the endoscope can be bent along two orthogonal directions using the navigation wheels (see fig. 1). One or two working channels going through the endoscope body allow to convey flexible endoscopic instruments. In order to navigate

Authors are with LSIIT (UMR CNRS-UDS 7005), Strasbourg University, France. inside the human body and to guide the instruments, the physician uses the images transmitted by an optical system (CCD camera), embedded at the tip of the endoscope.

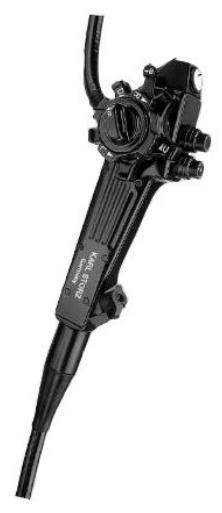

(a)

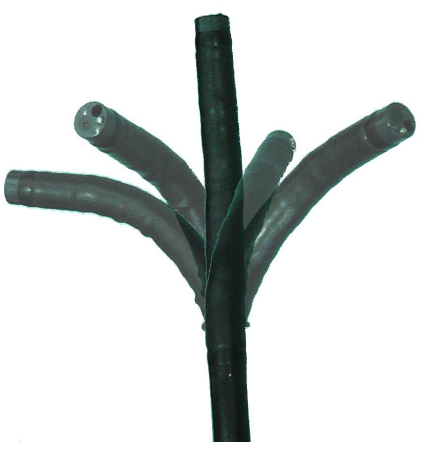

(b)
Fig. 1. Control handle (a) and bending tip of a flexible endoscope in different positions (b).

Surgeons face numerous challenges when handling flexible endoscopes and several difficulties have been identified:

- The flexible shaft of the endoscope cannot be directly controlled. Its shape is defined by anatomical constraints i.e. the anatomical structures which are in contact with the endoscope, e.g. the oesophagus or the stomach. Therefore the shape is generally unknown for the surgeon. Hence, the actions upon the endoscope body at the proximal end have hardly predictible effects on the endoscopic image (see fig. 2).

- Multiple actions have to be combined to perform the desired movements.

- Physiological motions of the organs due to breathing generate critical disturbances on the flexible endoscope. An expert hand-eye coordination is required to operate despite these disturbances.

- Classical endoscopic instruments are not articulated. The surgeon can only push or pull them inside the endoscope working channels, resulting in a translation along the direction of the tip of the endoscope. The surgeon must then move the tip of the endoscope in order to drive the instruments in other directions.

For NOTES procedures, the high number of mobilities to manage implies that several physicians simultaneously handle the endoscopic device and its instruments. Hence, they have to share a small working space around the endoscope control 


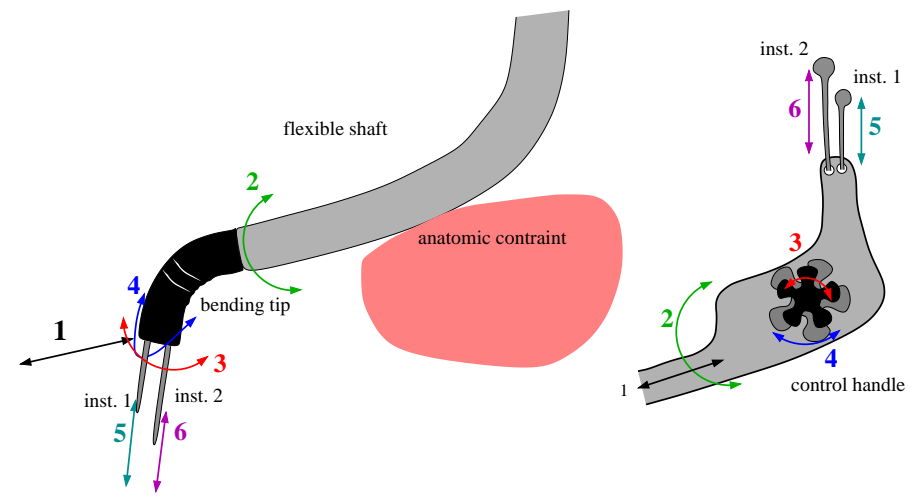

Fig. 2. Transmission of motions between the endoscope handle (right) and the endoscope bending tip (left). The effects of the translation (1) and rotation (2) of the endoscope depend on the flexible shaft shape which is defined by the anatomic constraints and which is generally unknown.

handle, and an excellent coordination is needed.

We are mainly interested in helping the surgeon in performing accurate medical tasks once on the operating area. Around the operating area, the endoscopist primilarly uses the navigation wheels to orient the endoscope towards the anatomical target, and the instruments to perform the surgical task. If the tip of the endoscope can be virtually linked to the anatomical target despite the physiological motions, the physician would be able to focus on the manipulation of the endoscopic tools. Therefore, we propose to partially robotize the motion of the endoscope in order to improve the manipulability of the endoscope and to automatically stabilize it with respect to anatomical targets despite their motion.

Physiological motion compensation using robots is a promising approach for assisting surgeons [15]. It has been used in laparoscopic surgery for compensating breathing motions [8] and in cardiac surgery for stabilizing the surgical instruments with respect to the beating heart [1]. But physiological motion rejection has never been applied to flexible endoscopes. The main difference arises from the fact that the camera is embedded at the tip of the endoscope in an eye-inhand configuration. As a consequence, physiological motion and self-motion of the endoscope cannot be easily separated.

In the presented work, the virtual link between the tip of the endoscope and the anatomical target is performed using visual servoing techniques. Indeed, vision is the only exteroceptive sensor naturally embedded in the flexible endoscope.

The paper is organized as follows. The second section presents how the robotized endoscope has been designed. Section III introduces the mechanical and vision model of the flexible endoscopic system. The proposed control algorithms for periodical motion rejection using visual servoing are presented in section IV with a stability analysis. Finally, in vitro and in vivo results are presented in section $\mathrm{V}$. The paper concludes with a discussion on the results, limitations of the current system and work in progress ${ }^{1}$.

\section{ENDOSCOPE ROBOTIZATION}

In conventional endoscopes, the control wheels are mounted on two coaxial shafts which drive two pulleys. Two pull-wires encircle the pulleys, run all the way through the endoscope shaft and are attached to the bending tip. When rotating the wheels, the distribution of the length of the wire on each side of the shaft is modified, which creates the bending of the tip (see fig. 6).

For improving the manipulability of the endoscope once on the operating area, we have chosen to motorize the deflection of the tip of the endoscope.

\section{A. Endoscope motorization}

Our solution for motorizing the endoscope consists in minimizing the modification to conventional endoscopes because these systems have been optimized for allowing large workspaces and good resistance to efforts. Our robotic endoscope experimental prototype is based on a single working channel flexible gastroscope Karl Storz 13801PKS. This endoscope has a diameter of $9.5 \mathrm{~mm}$ and is $1 \mathrm{~m}$ long. It can be used in a large variety of interventions including gastroscopy, transgastric and transvaginal procedures. The navigation wheels have been replaced by two hollow shaft rotary motors which drive the two coaxial shafts coming out from the handle (see fig. 3). By plugging hollow shaft motors directly on the shafts, it is possible to drive the system without adding gears in the assembly (see fig. 3). In this way, additional backlash is not introduced in the system. Motors have been chosen to guarantee a sufficient torque to reach extremal positions of the workspace and to minimize the weight of the system. Harmonic Drive FHA-8C motors, with a 50 reduction ratio and equipped with incremental encoders, have been selected. The motors are velocity controlled by built-in loops running on Harmonic Drive SC-610 servo controllers.

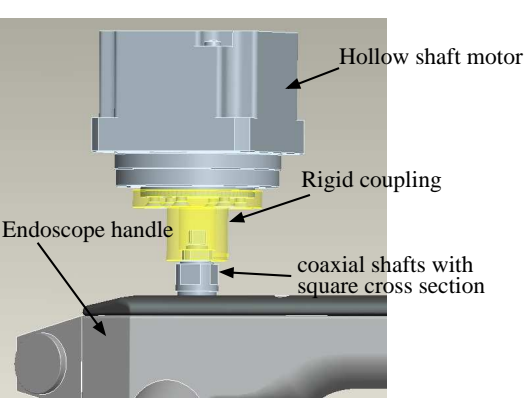

(a)

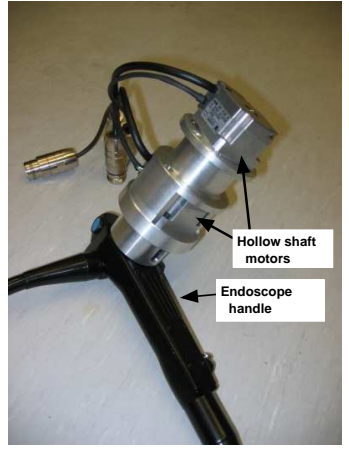

(b)
Fig. 3. CAD view of the motor / endoscope coupling (a) and real view of the handle of the motororized endoscope (b).

\footnotetext{
${ }^{1} \mathrm{~A}$ part of this work concerning the basic repetitive control of the endoscope has been published in [12]. However, backlash was not taken into account and gains of the controller were tuned by hand. This paper presents for the first time many improvments over this former paper, namely the automatic estimation of gain, the backlash compensation procedure and an analysis of the repetitive controller stability.
} 


\section{B. Control of the robotized flexible endoscope}

The control scheme of the robotized endoscope is shown on fig. 4. A position loop, which uses encoders measures and runs at $500 \mathrm{~Hz}$ on a PC-based controller, allows to compensate backlash (see section IV-D). The maximum output speed (120 $\mathrm{rpm})$ enables to cover the entire workspace, i.e. a half-turn of the wheel for each axis, in a quarter of a second which is sufficient for conventional endoscopic interventions.

Images provided by the embedded camera are transmitted to a display screen for visual feedback to the physicians and to the PC-based controller via a specific acquisition unit (Telecam SLII from Karl Storz) which can apply preprocessing of the image, like noise filtering. For visual servoing purposes, an external visual loop is running on the computer at the frame rate $(25 \mathrm{~Hz})$.

The robotized endoscope can be velocity controlled by the surgeon along the two motorized DOFs using a conventional joystick.

\section{Visual control principle}

In addition to classical control modes, a high-level automatic feedback mode using visual servoing has been added to the system. The objective of this mode is to automatically focus the endoscope on an anatomical target (see fig. 4). Let assume for now that the target can be selected and tracked in the endoscopic image. The visual servoing control scheme is shown on figure 9. Since generally no model of the target is available, an image-based visual servoing scheme has been selected. By clicking a position in the image, the user can provide the reference for the position of the target in the image. In the case of a static target, classical visual servoing techniques [4] allow to servo to zero the error e between the reference given by the user $\mathbf{r}$ and the position measured in the image $\mathbf{y}$. However, for a moving target, due to delays in the feedback loop, conventional controllers do not allow to simultaneously guarantee good stability margins and small tracking errors. For solving this problem, a repetitive controller will be used as described in section IV.

\section{Modeling OF THE SYSTEM}

A model of the endoscope is necessary for visual servoing purposes. It is especially important in the case of the use of a repetitive controller as it will be shown in section IV. The kinematic model of the motorized endoscope is presented in this section. The endoscope bending tip is composed of many stacked vertebrae and can be considered as a continuum robot. Classical methods for kinematic modeling cannot be directly applied as for discrete joints robots. In [9], the continuum robot is modeled using a modified Denavit-Hartenberg approach with interdependent parameters. Here, we briefly provide a direct geometric approach to obtain the kinematic model of the bending tip of the endoscope.

\section{A. Forward position kinematics}

The link between the motors and the wires is ensured by pulleys of radius $R_{p}$. The wires are attached at equally spaced

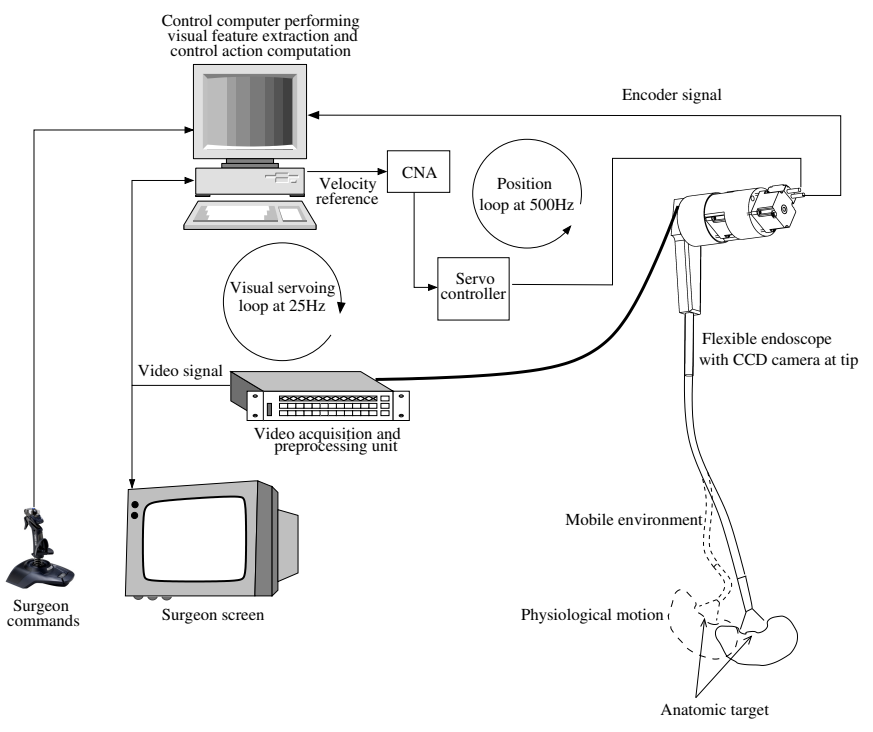

Fig. 4. The robotized endoscope control scheme

positions on the bending tip circumference (see fig. 6). For modeling, the wires and the continuum section are assumed inextensible.

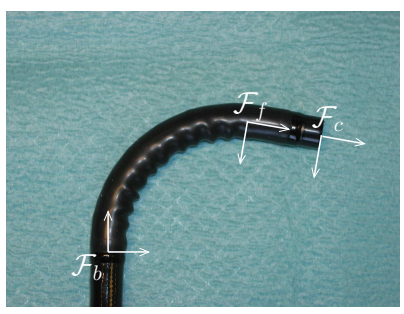

Fig. 5. Frames associated to the bending tip of the flexible endoscope

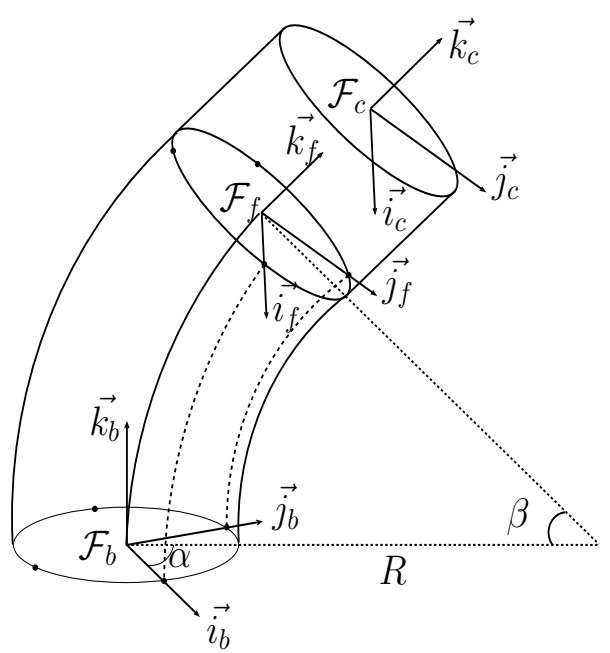

Fig. 6. Perspective view of the bending tip

The bending tip is composed of a flexible section of backbone length $L_{f}$ and of a rigid section at the extremity of the bending tip. The endoscopic camera is embedded at the extremity of the rigid section at distance $L_{t}$ from the flexible section. $\mathcal{F}_{b}$ is attached to the basis of the bending tip, with 


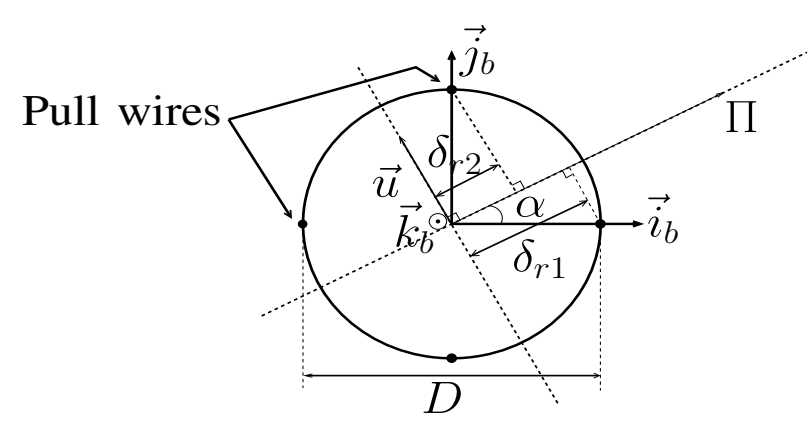

Fig. 7. Top view of the bending tip basis

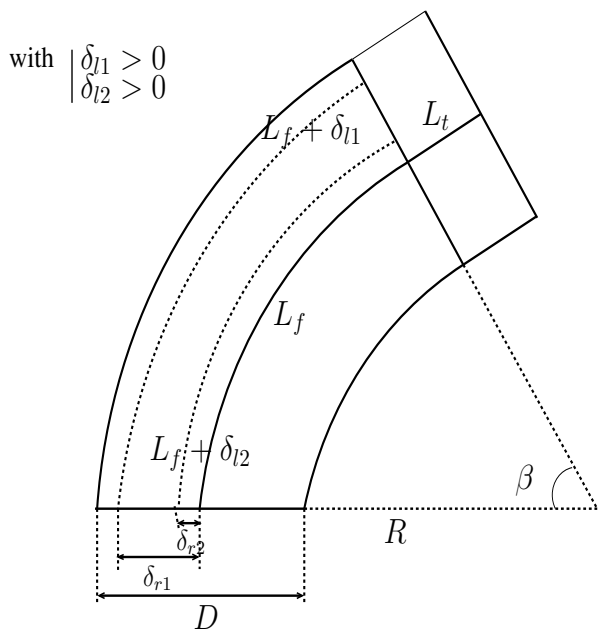

Fig. 8. Orthogonal projection in the bending plane $\Pi$

$\vec{k}_{b}$ along the backbone of the bending section and with $\vec{i}_{b}$ and $\vec{j}_{b}$ pointing towards pull wires (see fig. 6) and $\mathcal{F}_{c}$ is attached to the endoscopic camera with $\vec{k}_{c}$ perpendicular to the image plane and $\vec{i}_{c}$ along the $x$ axis of the image.

We aim at controlling the pose $\mathbf{r}$ of the endoscopic camera expressed in the frame $\mathcal{F}_{b}$. Hence we are interested in the relationship between the rotary motors positions $\mathbf{q}=\left[\begin{array}{ll}q_{1} & q_{2}\end{array}\right]^{\mathrm{T}}$ and the translation $\mathbf{t}_{b \rightarrow c}$ and the rotation $\mathbf{R}_{b \rightarrow c}$ from the base frame $\mathcal{F}_{b}$ to the camera frame $\mathcal{F}_{c}$.

Let call $\Pi$ the plane containing the endoscope backbone. $\alpha$ is the angle between the base frame and $\Pi$, and $\beta$ is the bending angle in $\Pi$. For the considered endoscope $\mathcal{F}_{c}$ and $\mathcal{F}_{b}$ are parallel when the bending tip is straight, i.e. the image plane is perpendicular to the backbone of the bending tip and the pull wires in the bending tip are aligned with the image axes of the endoscopic camera. ${ }^{2}$

With this assumption, the rotation matrix $\mathbf{R}_{b \rightarrow c}$ is defined by an angle $\beta$ around the normal $\mathbf{u}$ to the plane $\Pi$ (cf fig. 6 and 7):

$$
\mathbf{R}_{b \rightarrow c}=\left(\begin{array}{ccc}
s^{2} \alpha+c \beta c^{2} \alpha & -s \alpha c \alpha(1-c \beta) & c \alpha s \beta \\
-s \alpha c \alpha(1-c \beta) & c^{2} \alpha+c \beta s^{2} \alpha & s \alpha s \beta \\
-c \alpha s \beta & -s \alpha s \beta & c \beta
\end{array}\right)
$$

and the translation (cf fig. 8) can be expressed as

\footnotetext{
${ }^{2}$ Other configurations can be taken into account without necessity to reconsider the following of the paper.
}

$$
\mathbf{t}_{b \rightarrow c}=\left(\begin{array}{c}
L_{t} c \alpha s \beta+\frac{L_{f}}{\beta}(1-c \beta) c \alpha \\
L_{t} s \alpha s \beta+\frac{L_{f}}{\beta}(1-c \beta) s \alpha \\
L_{t} c \beta+\frac{L_{f}}{\beta} s \beta
\end{array}\right)
$$

where $s x$ and $c x$ respectively denote $\sin (x)$ and $\cos (x)$.

At rest $\left(\mathbf{q}=\left[\begin{array}{ll}q_{10} & q_{20}\end{array}\right]^{\mathrm{T}}\right)$, when no forces are applied on the wires, the bending part of the endoscope is straight. A rotation of angle $\delta_{q i}=q_{i}-q_{i 0}$ of the pulley $p_{i}$ acts on the wire $w_{i}$ and modifies the distribution of the length along the flexible part, so that the length difference with respect to rest is given by:

$$
\delta_{l i}=R_{p} \delta_{q i}, \forall i \in\{1,2\} .
$$

The length distribution of the two wires $\delta_{l 1}$ and $\delta_{l 2}$ can be related to the angles $\alpha$ and $\beta$ using $\delta_{r 1}$ and $\delta_{r 2}$ as shown on Fig 7 and 8 so that:

$$
\beta=\frac{2 R_{p}}{D} \sqrt{\delta_{q_{1}}^{2}+\delta_{q_{2}}^{2}}
$$

and

$$
\alpha= \begin{cases}0 & \text { if } \delta_{q_{1}}=\delta_{q_{2}}=0 \\ \operatorname{atan} 2\left(-\delta_{q_{2}},-\delta_{q_{1}}\right) & \text { otherwise }\end{cases}
$$

Finally the kinematic model $\mathbf{T}_{b \rightarrow c}\left(q_{1}, q_{2}\right)$ can be obtained from equations (1), (2), (4), (5).

\section{B. Forward differential kinematics}

Let define $\boldsymbol{\Theta}=[\alpha, \beta]^{T}$ and $\boldsymbol{\Delta} l=\left[\delta_{l 1}, \delta_{l 2}\right]^{T}$. The velocity screw of the camera $\mathbf{V}=[\mathbf{v}, \omega]^{T}$ expressed in $\mathcal{F}_{c}$ is related to the joint velocity $\dot{\mathbf{q}}$ by the robot Jacobian $\mathbf{J}_{\mathbf{q}}$ :

$$
\mathbf{V}=\mathbf{J}_{\mathbf{q}} \dot{\mathbf{q}} \text { with } \mathbf{J}_{\mathbf{q}}=\mathbf{J}_{\Theta} \frac{\partial \boldsymbol{\Theta}}{\partial \mathbf{q}}
$$

where

$$
\frac{\partial \boldsymbol{\Theta}}{\partial \mathbf{q}}=\left(\begin{array}{cc}
-\frac{\delta_{q 2}}{\delta_{q 1}^{2}+\delta_{q 2}^{2}} & \frac{\delta_{q 1}}{\delta_{q 1}^{2}+\delta_{q 2}^{2}} \\
\frac{2 R_{p}}{D} \frac{\delta_{q 1}}{\sqrt{\delta_{q 1}^{2}+\delta_{q 2}^{2}}} & \frac{2 R_{p}}{D} \frac{\delta_{q 2}}{\sqrt{\delta_{q 1}^{2}+\delta_{q 2}^{2}}}
\end{array}\right) .
$$

$\mathbf{J}_{\Theta}$ which relates $\dot{\boldsymbol{\Theta}}$ to the camera velocity screw $(\mathbf{V}=$ $\left.\mathbf{J}_{\Theta} \dot{\Theta}\right)$ is obtained as

$\mathbf{J}_{\Theta}=\left(\begin{array}{cc}-\frac{L_{f}}{\beta} s \alpha(1-c \beta)-L_{t} s \alpha s \beta & \frac{L_{f}}{\beta^{2}} c \alpha(1-c \beta)+L_{t} c \alpha \\ \frac{L_{f}}{\beta} c \alpha(1-c \beta)+L_{t} c \alpha s \beta & \frac{L_{f}}{\beta^{2}} s \alpha(1-c \beta)+L_{t} s \alpha \\ 0 & \frac{L_{f}}{\beta}\left(1-\frac{s \beta}{\beta}\right) \\ -c \alpha s \beta & -s \alpha \\ -s \alpha s \beta & c \alpha \\ -1+c \beta & 0\end{array}\right)$ 


\section{Model of the visual loop}

The model of the visual loop is given in fig. 9. The control action computed by the controller is a joint velocity reference $\dot{\mathbf{q}}^{*}$. It is used to compute a position reference for the position loop. The bandwidth of the joint position loops is largely higher than the Nyquist frequency of the visual loop $(12.5 \mathrm{~Hz})$. Hence, the dynamic of the actuators can be safely neglected: The actual joint velocity equals the reference $\left(\dot{\mathbf{q}} \sim \dot{\mathbf{q}}^{*}\right)$ and the block diagram simplifies to the one shown on fig. 9 .

The velocity of the image features $\dot{\mathbf{F}}$ is related to the joint velocity $\dot{\mathbf{q}}$ by $\dot{\mathbf{F}}=\mathbf{J} \dot{\mathbf{q}}$ where $\mathbf{J}=\mathbf{L}_{\mathbf{d}} \mathbf{J}_{\mathbf{q}}$ is the $2 \times 2$ image Jacobian relating the joints velocities to the image features velocities. $\mathbf{L}_{\mathbf{d}}$ is the interaction matrix of the considered image features which relates the velocity screw of the camera to the velocity of the image feature in the image. For example, for a point of position $[X, Y]^{T}$ in the image plane and of $3 \mathrm{D}$ position $[x, y, d]^{T}$ with respect to the camera frame,

$\mathbf{L}_{\mathbf{d}}=\left(\begin{array}{cc}G_{x} & 0 \\ 0 & G_{y}\end{array}\right)\left(\begin{array}{cccccc}-\frac{1}{d} & 0 & \frac{X}{d} & X Y & -\left(1+X^{2}\right) & Y \\ 0 & -\frac{1}{d} & \frac{Y}{d} & 1+Y^{2} & -X Y & -X\end{array}\right)$

where $G_{x}$ and $G_{y}$ are the magnification factors of the camera.

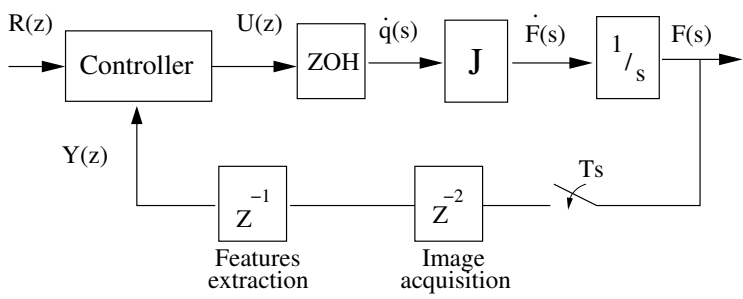

Fig. 9. Block diagram of the visual servoing loop

The discrete time open loop transfer function from the velocity reference and the measured position of the features in the image is then given by

$$
P(z)=\frac{Y(z)}{U(z)}=z^{-3} \mathcal{Z}\left\{Z O H \frac{\mathbf{J}}{s^{2}}\right\}=\mathbf{J} \frac{T_{s} \cdot z^{-4}}{1-z^{-1}}
$$

where $\mathcal{Z}$ represents the z-transform and $T_{s}$ is the sampling period of the loop. The delay $z^{-3}$ in the feedback loop accounts for the image acquisition and processing times.

Under the aforementioned assumptions, the dynamic part of the transfer function is constant. However, the gain matrix $\mathbf{J}$ depends on the position of the bending tip, on the position of the target in the image and on the depth $d$ of the target with respect to the camera.

\section{Model validation}

In order to compare our model to the actual behaviour of the flexible endoscope, one needs to measure the homogeneous transformation from the basis frame $\mathcal{F}_{b}$ to the camera frame $\mathcal{F}_{c}$.

We have chosen to use the images provided by the endoscopic camera to estimate the pose of the camera frame with respect to a calibrated environment composed of a box marked with black points on a white background. For allowing metric estimation, the endoscopic camera has been carefully calibrated using the method proposed in [18] and implemented in a toolbox by J. Bouguet [3].

For validating the model, the flexible shaft of the endoscope has been maintained in a fixed position with respect to the environment and the bending tip has been moved across the entire workspace. The pose of the camera with respect to the environment $\mathbf{T}_{e n v \rightarrow c}^{i}$ is estimated from each image $\mathcal{I}_{i}$ and the encoder positions $\mathbf{q}^{i}$ are recorded. The pose is obtained using the iterative algorithm proposed by Dementhon [6] and the Virtual Visual Servoing framework proposed by Marchand et al. [5].

Camera frame position, model prediction (red), measure (blue)

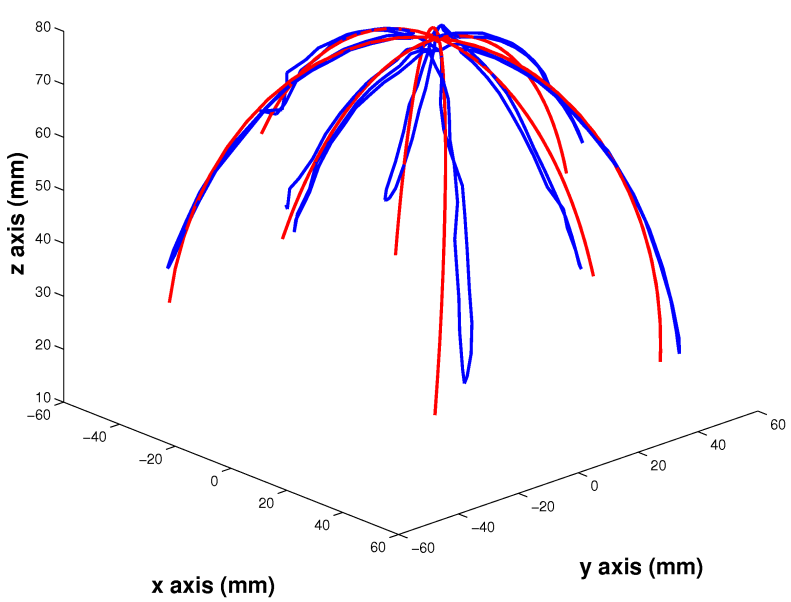

Fig. 10. View of the predicted and measured camera frame position expressed in the reference frame position

Fig. 10 shows the measured positions of the camera expressed in $\mathcal{F}_{b}$ compared with the positions predicted using the encoder positions and the forward kinematic model. The mean absolute distance error is $7.36 \mathrm{~mm}$ and the mean absolute orientation error is $11.93^{\circ}$. These errors should be compared to the size of the workspace, which is a surface close to a hemisphere of radius $70 \mathrm{~mm}$. The distances are computed in a point to point manner. If one considers instead the distance between the measured workspace surface of the endoscope and the workspace surface predicted using the kinematic model, the mean error drops to $2.78 \mathrm{~mm}$ with $1.99 \mathrm{~mm}$ standard deviation and $8.98^{\circ}$ in orientation with $3.41^{\circ}$ standard deviation.

The model provides a good representation of the workspace of the system. The errors account for several approximations: the endoscope is not a continuous system, there are non uniformities along the bending section, the camera is not exactly aligned with the endoscope axis and the wires in the bending tip. The difference between the geometric and point to point errors mainly accounts for the backlash in the system which are described in the next section. 


\section{E. Mechanical non linearities characterization}

Practically, flexible endoscopes have a complex non linear behaviour. The wire transmission between the navigation wheels and the bending tip induces friction which results in backlash. When changing the direction of motion on one of the joints, the motor motion has no effect on the endoscope tip while the joint position stays in a deadband. For characterizing the backlash, the motors have been submitted to forth-andback motions while measuring the position in the image of a static target. This motion has been repeated at different operating points in order to cover the workspace of the bending tip. It appears that the hysteresis shape (Fig.13) is not a pure backlash model. In addition to the deadband, the hysteresis curve presents a non linear segment before the linear part. From the hysteresis curve one can identify the hysteresis width and the deadband size. Fig 11 and 12 show the deadband measurement obtained from these motions. It can be seen that the deadband of the backlash depends on the position of the bending tip. Unfortunately, these backlash maps cannot be directly used because the deadband of the backlash also depends on the shape of the flexible shaft of the endoscope, which is generally unknown, as shown in fig. 13. In order to solve this problem, a method for estimating backlash in situ will be proposed in section $\mathrm{V}$.

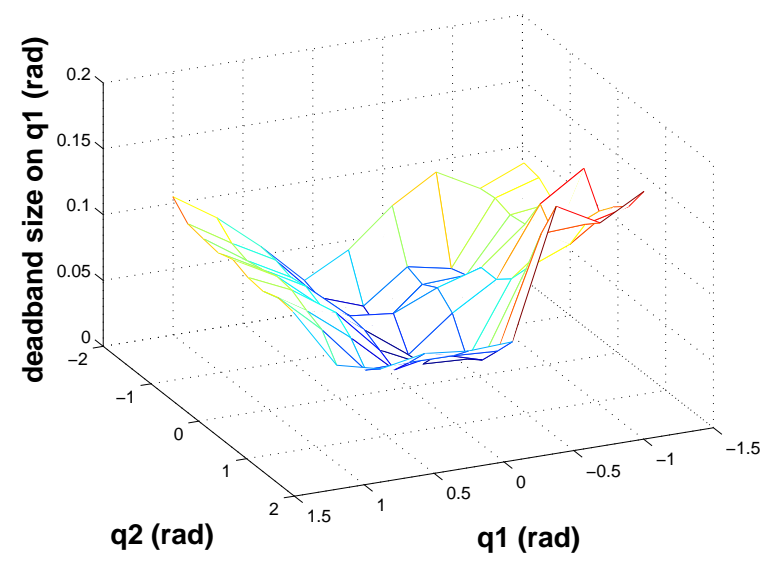

Fig. 11. Backlash hysteresis map for axis 1 . Only the first actuator is moving and the target position is measured on the $\mathrm{X}$ image axis.

\section{PERIODIC MOTIONS CANCELLATION}

Physiological motion, especially breathing, acts as an output disturbance on the position of the target in the image. When the patient is anaesthetized and placed under artificial ventilation (as for instance during transluminal interventions), the cycle of the respiration is periodical. For example, the spectrum analysis of the liver motion of a pig under artifical ventilation (fig. 14) clearly shows that the motion is periodic.

\section{A. Repetitive control}

In the context of surgical robotic, the robotized endoscopic system must be able to reject periodic disturbances and to follow the surgeons commands provided as reference image

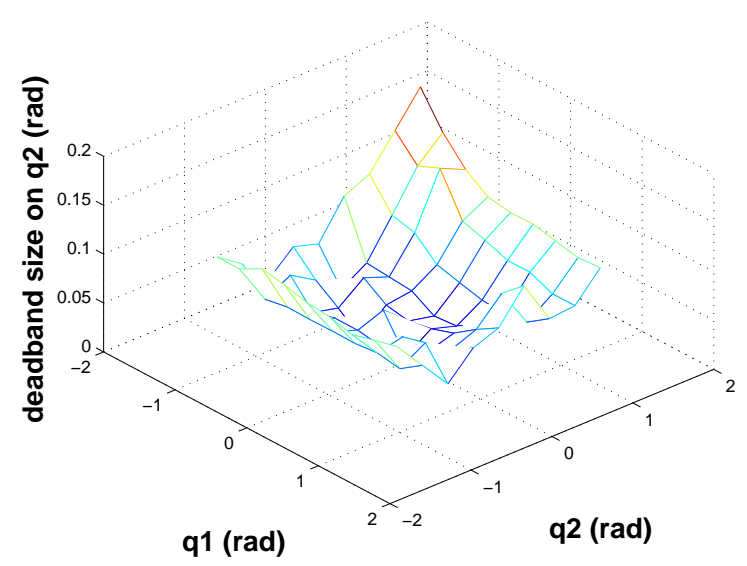

Fig. 12. Backlash map for axis 2. Only the second actuator is moving and the target position is measured on the $\mathrm{Y}$ image axis.

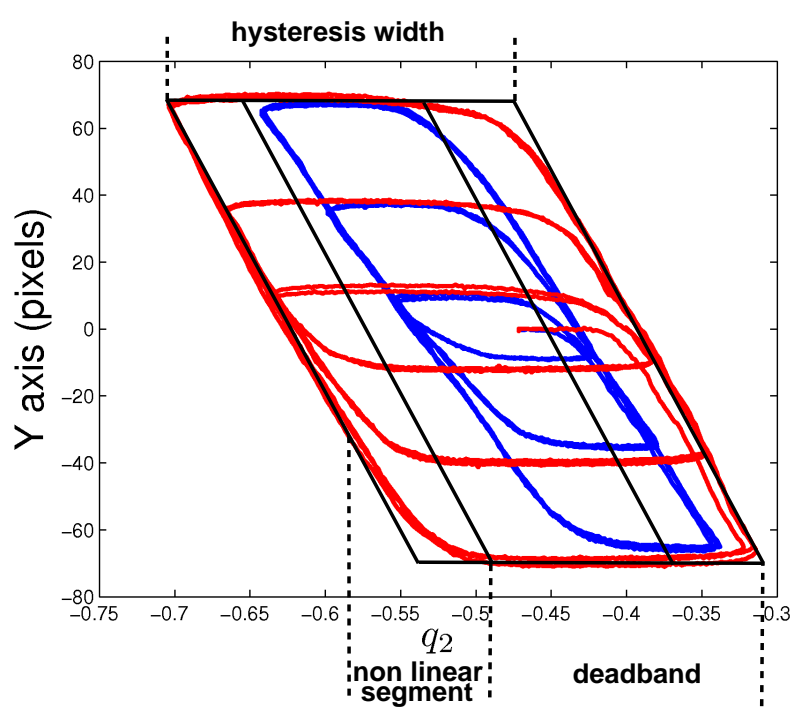

Fig. 13. Backlash hysteresis for axis 2 at the same joint position for two different shapes of the flexible shaft of the endoscope. The hysteresis has smooth non-linearities, so that the hysteresis width (0.23 rad.) is different from the deadband width (0.12 rad.).

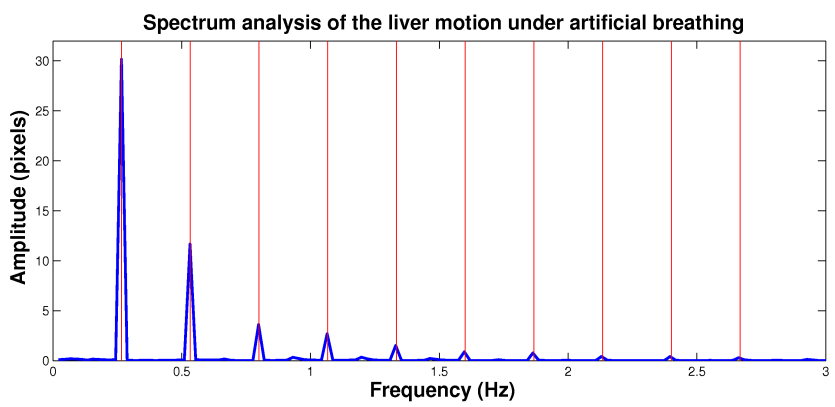

Fig. 14. Spectrum analysis of the motion of the liver of a pig under artificial ventilation obtained from an image sequence analysis. The fondamental frequency is $0.267 \mathrm{~Hz}$ and the Nyquist frequency which is $12.5 \mathrm{~Hz}$. No significant signal appears beyond $3 \mathrm{~Hz}$. 
positions. This is especially important in our application since the endoscopic instruments are not articulated and the endoscope must be moved to reach different points of interest. For this purpose, repetitive control with feedforward is used. Repetitive control is based on the internal model principle [7] that states that the inclusion of the model of a signal in a stable closed-loop system can assure perfect tracking or complete rejection of this signal. For rejecting periodic disturbances of period $T$, the controller has the following form

$$
\mathbf{C}_{r}\left(z^{-1}\right)=\frac{Q\left(z, z^{-1}\right) z^{-N}}{1-Q\left(z, z^{-1}\right) z^{-N}} \mathbf{L}\left(z, z^{-1}\right)
$$

with $N=\frac{T}{T_{s}}$ and where $Q\left(z, z^{-1}\right)$ is a low pass filter added for stability robustness purposes. $\mathbf{L}\left(z, z^{-1}\right)$ must be chosen for guarantying stability and performance (see section IV-B).

The repetitive control law is then given by the following equation

$$
\mathbf{u}_{r}[k]=Q\left(q, q^{-1}\right)\left(\mathbf{u}_{r}[k-N]+\mathbf{L}\left(q, q^{-1}\right) \mathbf{e}[k-N]\right)
$$

where $q^{-1}$ is the unit delay operator. The control action is composed of the repetition of the control action at the previous period of the disturbance and of a bettering term computed from the error signal $\mathbf{e}[k]$.

The basic form of the repetitive controller does not allow to follow references efficiently [12]. To track non periodic references, a feedforward controller built on a simulated model of the plant $\hat{\mathbf{P}}\left(z^{-1}\right)$ is added to the system. For our system, $\hat{\mathbf{P}}\left(z^{-1}\right)=\hat{\mathbf{J}} \frac{T_{s} z^{-4}}{1-z^{-1}}$, where $\hat{\mathbf{J}}$ is an estimate of the image Jacobian. Since the model of the plant contains an integrator, a proportional control law is used for the feedforward controller: $\mathbf{C}_{\text {ref }}\left(z^{-1}\right)=k_{c} \hat{\mathbf{J}}^{-1}$. By including $\hat{\mathbf{J}}^{-1}$ in the controller, the gain of the controller $k_{c}$ can be tuned independently of the configuration of the system.

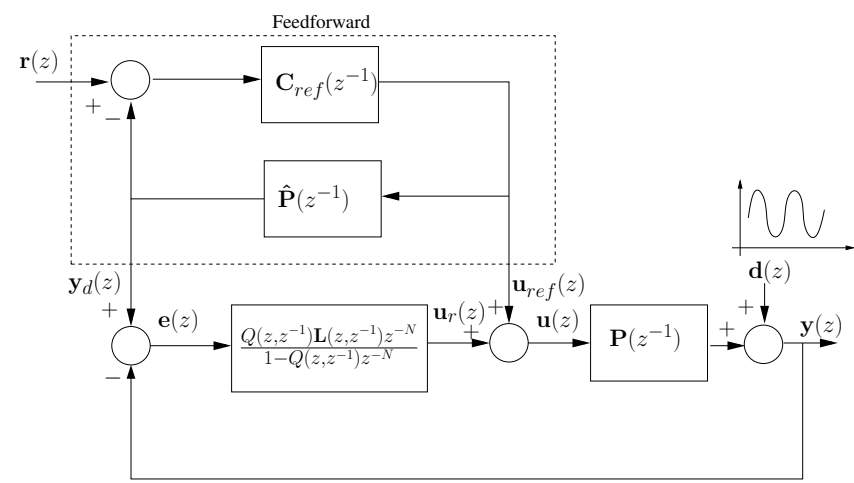

Fig. 15. Bloc diagram of the repetitive controller with reference tracking using a feedforward controller.

\section{B. Tuning the repetitive controller for stability and perfor- mances purposes}

For a steady reference, the closed loop error signal can be expressed the following manners:

$$
\begin{aligned}
\mathbf{e}(z)= & Q\left(z, z^{-1}\right)\left(\mathbf{I}-\mathbf{P}\left(z^{-1}\right) \mathbf{L}\left(z, z^{-1}\right)\right) z^{-N} \mathbf{e}(z) \\
& -\left(1-Q\left(z, z^{-1}\right) z^{-N}\right) \mathbf{d}(z)
\end{aligned}
$$

or

$$
\begin{aligned}
\mathbf{e}(z)= & -\left[\mathbf{I}+Q\left(z, z^{-1}\right) z^{-N}\left(\mathbf{P}\left(z^{-1}\right) \mathbf{L}\left(z, z^{-1}\right)-\mathbf{I}\right)\right]^{-1} \\
& \left(1-Q\left(z, z^{-1}\right) z^{-N}\right) \mathbf{d}(z) .
\end{aligned}
$$

a) Stability condition: Using the small-gain theorem it can be stated from Eq.(9) that if $Q$ and $\mathbf{P} L$ do not contain poles outside the unit circle and if

$$
\begin{gathered}
\left\|Q\left(z, z^{-1}\right)\left(\mathbf{P}\left(z^{-1}\right) \mathbf{L}\left(z, z^{-1}\right)-\mathbf{I}\right)\right\|_{\infty}<1 \\
\text { with } z=e^{j \omega T_{s}} \forall \omega T_{s} \in[0, \pi]
\end{gathered}
$$

then the closed loop is stable.

b) Disturbance rejection: From eq. 8 it appears that the free response error becomes null in one period if $\mathbf{P}\left(z^{-1}\right) \mathbf{L}\left(z, z^{-1}\right)=\mathbf{I}$. Moreover, from eq. 9, it can be seen that if $\left(1-Q\left(e^{j \omega T_{s}}, e^{-j \omega T_{s}}\right) e^{-j \omega N T_{s}}\right)=0$ for all harmonic frequencies of the disturbances then the periodic disturbances are perfectly rejected.

c) Controller tuning: Given the stability and disturbance rejection conditions, it is interesting to choose $\mathbf{L}\left(z, z^{-1}\right)$ as the inverse of the plant model $\hat{\mathbf{P}}\left(z^{-1}\right)$ [17]. This is possible for stable minimum phase systems. If the plant is unstable, the repetitive controller can be applied to the stabilized plant. More complex solutions have been proposed for unstable ([17]) and non minimum phase systems ([17],[13]).

It is difficult to obtain a model capturing all the dynamics of the plant. From Eq.(10), it can be seen that the stability condition is relaxed if the filter $Q\left(z, z^{-1}\right)$ has a low gain at frequencies where the model is uncertain. However, for obtaining small residual errors $\left(1-Q\left(e^{j \omega T_{s}}, e^{-j \omega T_{s}}\right) e^{-j \omega N T_{s}}\right)$ should equal zero at the harmonic frequencies $\omega=k \frac{2 \pi}{N T_{s}}$, for $k=$ $1, \ldots, \frac{N}{2}$ of the disturbance. $Q\left(z, z^{-1}\right)$ is usually chosen as a non causal zero phase low pass filter with a unit DC gain which satisfies a compromise between stability robustness and performance.

In our application, the plant model contains a pole in 1 and does not contain any unstable zeros. It is possible to directly use the invert of the plant model

$$
\mathbf{L}\left(z, z^{-1}\right)=\hat{\mathbf{P}}^{-1}\left(z^{-1}\right)=\hat{\mathbf{J}}^{-1} \frac{1-z^{-1}}{T_{s} z^{-4}}
$$

because $\mathbf{C}_{r}\left(z^{-1}\right)$ also contains a pole in 1 , so that finally no unstable pole/zero compensation occurs between the controller and the plant.

The filter has been tuned as $Q\left(z, z^{-1}\right)=0.35+0.24\left(z^{-1}+\right.$ $z)+0.075\left(z^{-2}+z^{2}\right)+0.01\left(z^{-3}+z^{3}\right)$ which corresponds to a $2.93 \mathrm{~Hz}-3 \mathrm{~dB}$ cut off frequency low pass filter. Indeed, as shown on fig. 14 there is no significant harmonics in breathing motion above $3 \mathrm{~Hz}$. Considering that $Q$ handles uncertain 
dynamics, the stability condition Eq.(10) is then given by the error on the Jacobian estimation as follows

$$
\left\|\mathbf{J} \hat{\mathbf{J}}^{-1}-\mathbf{I}\right\|_{2}<1
$$
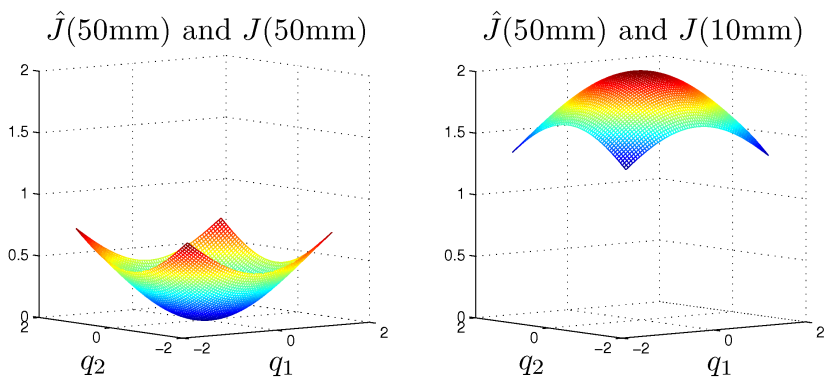

$\hat{J}(10 \mathrm{~mm})$ and $J(50 \mathrm{~mm})$
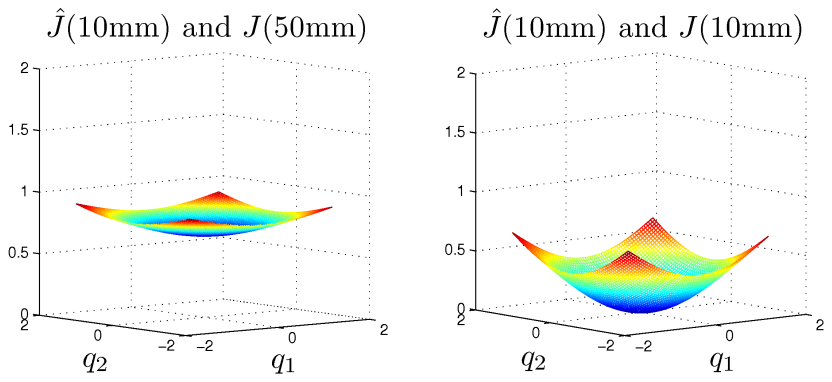

Fig. 16. $\left\|\mathbf{J} \hat{\mathbf{J}}^{-1}-\mathbf{I}\right\|_{2}$ where the estimated Jacobian $\hat{\mathbf{J}}$ is evaluated for a point on the optical axis at two different depths, i.e. $X, Y=0$ and $d=10 \mathrm{~mm}$ or $50 \mathrm{~mm}$, at the tip straight position, i.e. $q_{1}, q_{2}=0$. The real Jacobian is evaluated for each joint position for a point on the optical axis at two different depths, i.e. $X, Y=0$ and $d=10 \mathrm{~mm}$ or $50 \mathrm{~mm}$.

Fig. 16 shows the value of $\left\|\mathbf{J} \hat{\mathbf{J}}^{-1}-\mathbf{I}\right\|_{2}$ on the workspace of the bending tip for a point on the optical axis (i.e. $X=Y=0$ ) at two different depths $(d=10 \mathrm{~mm}$ or $50 \mathrm{~mm})$ when $\hat{\mathbf{J}}$ is estimated for the bending tip in straight position (i.e. $q_{1}=q_{2}=0$ ) for a point on the optical axis (i.e. $X=Y=0$ ) at the same depths. These curves show the need of estimating the jacobian of the system, since using a fixed jacobian does not allow to guarantee stability. Especially, the sufficient condition for stability can be invalidated when the depth is overestimated.

\section{Simulations}

Fig. 17 and 18 present simulation results of the proposed repetitive controller with $k_{c}=3.5$. This gain ensures a minimum $5 \%$ settling time $(0.5 \mathrm{~s})$ with a $10 \mathrm{~dB}$ gain margin and $60^{\circ}$ phase margin. The motion of the target is purely sinusoidal with frequency $0.2 \mathrm{~Hz}$ and a $1 \mathrm{~cm}$ amplitude. In the rest position $\left(q_{1}=\frac{\pi}{3}, q_{2}=\frac{\pi}{5}\right)$ and the depth of the target is $d=50 \mathrm{~mm}$. Fig. 17 shows the behaviour of the loop when the jacobian matrix is estimated at the rest position: $\left(\hat{\mathbf{J}}\left(z^{-1}\right)\left[\begin{array}{ll}-983 & -115 \\ -113 & -841\end{array}\right]^{3}\right)$. The disturbance is very well rejected in only one period and the reference is tracked as expected. A very small error appears after the reference step due to the modification of the system model. Fig. 18 shows the results for the same configuration of the endoscope but when the used image Jacobian has been estimated at the

\footnotetext{
${ }^{3}$ All jacobian matrix elements are expressed in pixels $/ \mathrm{mm}$.
}

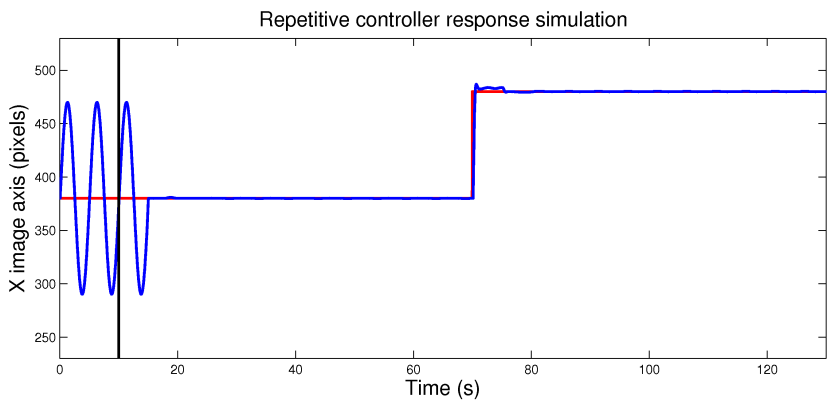

Fig. 17. Simulation of the proposed controller tuned with the actual depth of the target. The controller is activated at $t=10 \mathrm{~s}$ and a reference step occurs at $t=70 \mathrm{~s}$.

actual actuator positions but for a point on the optical axis at depth $d=10 \mathrm{~mm}: \hat{\mathbf{J}}=\left[\begin{array}{cc}-2915 & -257 \\ -251 & -2583\end{array}\right]$. As expected from the previous analysis, the modeling error does not lead to instability. However, the performance of the controller for rejecting disturbances decreases drastically and many periods are needed to learn the disturbance. The feedforward controller is also not able to cancel the reference tracking error and the repetitive controller needs several iterations to compensate the residual error.

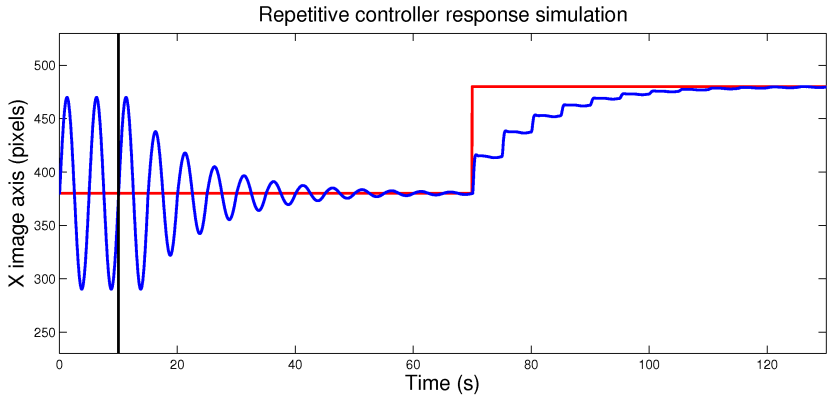

Fig. 18. Simulation of the proposed controller tuned with an error on the depth.The controller is activated at $t=10 \mathrm{~s}$ and a reference step occurs at $t=70 s$.

\section{Backlash compensation strategy}

Pure backlash can be compensated by inverting the backlash model [16]. When direction is inverted on one of the axis, a motion of amplitude $d_{b}$ is added to the reference. This procedure can be simply handled in the position loop of the motor. However, since the deadband of the backlash depends on the shape of the endoscope, we cannot identify the deadband size map offline and apply the corresponding backlash inverse model. An in situ estimation of the backlash deadbands is then required.

As shown on fig. 13, the hysteresis curves on the endoscopic system are complex and several backlash compensation strategies can be used. In order to decide which value should be used to set the deadband $d_{b}$ of the backlash inverse compensation, three strategies have been compared. The simulation results are presented on fig. 19. In the first strategy no backlash compensation is used, in the second the deadband width $(0.15$ 
rad.) is used, whereas the hysteresis width ( $0.2 \mathrm{rad}$.$) is used$ in the third. The controller has been tuned with an accurate estimate of the Jacobian.

After convergence, the maximum absolute error and the mean absolute error are respectively 3.76 pix. and 0.31 pix. without backlash compensation, 0.62 pix. and 0.11 pix. with $d_{b}$ set to the deadband width and 6.19 pix. and 0.41 pix. with $d_{b}$ set to the hysteresis width. Without backlash compensation, the repetitive controller learns the residual error due to backlash and allows to decrease it over time. However, because of the $Q$ filter, the disturbance cannot be completely rejected. The backlash compensation using the identified hysteresis width avoids the occurence of the reference repetition, but it also results in periodic jolts and induces a greater residual error after convergence than without any backlash compensation. Finally, the best strategy is to apply the backlash inverse with the deadband width. This allows to reduce both the repetition of the reference and the residual error after convergence.
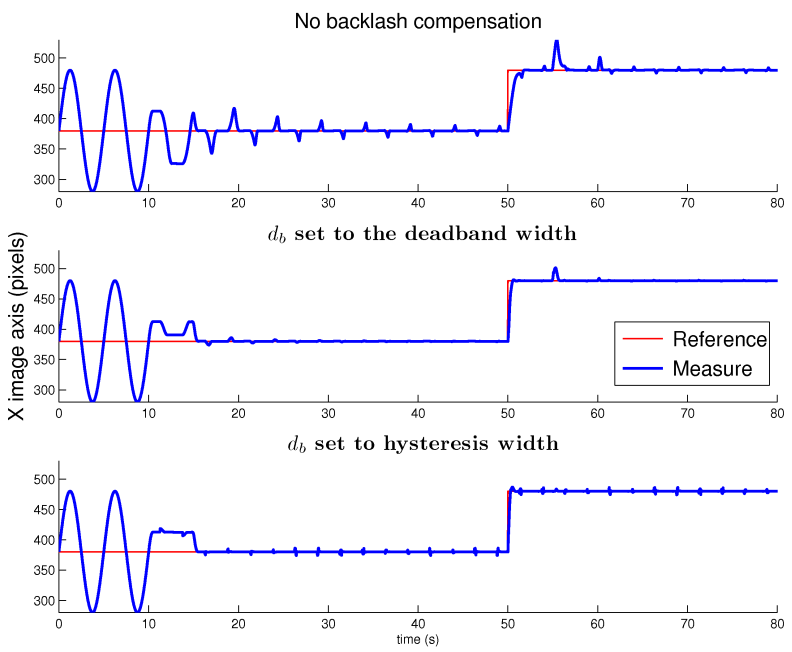

Fig. 19. Comparison of the backlash compensation strategies.

\section{EXPERIMENTAL RESULTS}

Since the depth intervening in the model of the system is generaly not available, we propose to estimate the jacobian of the system. The method of estimation as well as the practical method for deadband estimation are explained in this section. This section also presents experimental results obtained in vitro and in vivo.

\section{A. Visual features}

For servoing the 2DOFs of the bending tip, a single image point (two coordinates) is sufficient as visual feature. However, the environment of our application is complex without the possibility to add fiducials. The solution proposed here lies on template matching algorithms. The area of interest is first manually selected by the surgeon and is learned as the reference template. For each new image, the parameters of the transformation (translation, affine, homographic, etc.) which minimize a dissimilarity measure between the current image and the reference template (usually a sum of pixel to pixel squared differences) are searched using a local optimization algorithm. These techniques provide an estimate of the transformation between the area of interest in the current image and its position in the initial image, so that any point from the initial area of interest can be used as a visual feature.

In the following in vitro and in vivo experiments, the visual features $\mathbf{F}$ are the coordinates of the center of the window defined by the user. The window is tracked using the ESM tracking algorithm [2], which estimates homography transformations. It is well suited for tracking small planar patches and proved to be quite robust under in vivo conditions.

\section{B. Jacobian estimation}

It is difficult to estimate the Jacobian matrix from the model detailed in section III because the depth of the target in the camera frame is generally unknown. Depth could be estimated using structure from motion techniques. However, since in situ estimations have to be involved, we prefer to directly estimate the Jacobian matrix. Therefore, we propose an automatic direct estimation of the gains of the Jacobian matrix. Such an estimation includes the magnification factors of the camera and also avoids the need of camera calibration.

On a static environment, the image velocities are related to the joint velocities by the following equation

$$
\dot{\mathbf{F}}=\left[\begin{array}{ll}
J_{x 1} & J_{x 2} \\
J_{y 1} & J_{y 2}
\end{array}\right] \dot{\mathbf{q}}
$$

where $J_{x 1}, J_{x 2}, J_{y 1}, J_{y 2}$ are the unknown gains of the Jacobian matrix. By performing at least two controlled displacements $\dot{\mathbf{q}}_{a}=\left[\dot{q}_{1 a}, \dot{q}_{2 a}\right]^{T}$ and $\dot{\mathbf{q}}_{b}=\left[\dot{q}_{1 b}, \dot{q}_{2 b}\right]^{T}$ and by measuring the corresponding image target velocities $\dot{\mathbf{F}}_{a}=\left[\dot{X}_{a}, \dot{Y}_{a}\right]^{T}$ and $\dot{\mathbf{F}}_{b}=\left[\dot{X}_{b}, \dot{Y}_{b}\right]^{T}$ it is possible to estimate the local Jacobian

$$
\left[\begin{array}{l}
J_{x 1} \\
J_{x 2} \\
J_{y 1} \\
J_{y 2}
\end{array}\right]=\left[\begin{array}{cccc}
\dot{q}_{1 a} & \dot{q}_{2 a} & 0 & 0 \\
\dot{q}_{1 b} & \dot{q}_{2 b} & 0 & 0 \\
0 & 0 & \dot{q}_{1 a} & \dot{q}_{2 a} \\
0 & 0 & \dot{q}_{1 b} & \dot{q}_{2 b}
\end{array}\right]^{-1}\left[\begin{array}{c}
\dot{X}_{a} \\
\dot{X}_{b} \\
\dot{Y}_{a} \\
\dot{Y}_{b}
\end{array}\right]
$$

The displacements should be independent for inversion purposes and large enough in order to obtain the image velocities out of the backlash non linear zones. We chose joint velocities $\dot{\mathbf{q}}_{a}=\Omega\left[\cos \left(\frac{\pi}{4}\right), \sin \left(\frac{\pi}{4}\right)\right]^{T}$ and $\dot{\mathbf{q}}_{b}=\Omega\left[\cos \left(\frac{3 \pi}{4}\right), \sin \left(\frac{3 \pi}{4}\right)\right]^{T}$, where $\Omega$ is constant.

Practically, the target has also its own motion which should be discriminated from the effect of the camera motion. For this purpose, we use the periodicity of the target self-motion. It is first recorded during one period of the disturbance while the endoscope bending tip is not activated. The corrected image position $\mathbf{F}_{c}$ during the endoscope motion is then obtained as:

$$
\mathbf{F}_{c}[k]=\mathbf{F}[k]-\mathbf{F}[k \bmod N],
$$

where $[k \bmod N] \in\{0, \cdots, N-1\}$ and $\mathbf{F}[k \bmod N]$ is the learned image position during the first period without endoscope motion. 


\section{Deadband estimation}

The hysteresis curve is obtained independently for each axis by performing open loop forth-and-back motions. For each axis, the effect of the motor motion is observed in the image along the main direction. The effect of the disturbance is removed in the same manner as for the Jacobian estimation. The deadband estimation is performed both at the top (red) and at the bottom (green) of the hysteresis curve (Fig. 20). The first step consists in finding the points of extreme abscissas (change of motor direction) $O_{t}=\left[x_{o t}, y_{o t}\right]^{T}$ and $O_{b}=$ $\left[x_{o b}, y_{o b}\right]^{T}$. Then, the points on the curve $P_{t}=\left[x_{p t}, y_{p t}\right]^{T}$ and $P_{b}=\left[x_{p b}, y_{p b}\right]^{T}$ with same ordinates and farthest from $O_{t}=\left[x_{o t}, y_{o t}\right]^{T}$ and $O_{b}=\left[x_{o b}, y_{o b}\right]^{T}$ are located. The deadbands are then estimated as

$$
d_{b t}=\left|x_{o t}-x_{p t}\right| \text { and } d_{b b}=\left|x_{o b}-x_{p b}\right| \text {. }
$$

Finally, we choose the smallest value of the identified deadbands $d_{b}=\min \left(d_{b b}, d_{b t}\right)$ to set the backlash inverse compensation in order to limit the jolts.

\section{Experiments on a laboratory Test Bed}

We have developed a laboratory testbed to validate the control strategy. We use a model of the abdominal cavity organs which is fixed to a motorized device, so that the motion of the target can be controlled to produce a periodic output disturbance.

In the presented experiment, the disturbance is a pure sinusoide of period $5 \mathrm{~s}$. The gains of the Jacobian matrix have been estimated as explained in section V-B and one found $\hat{\mathbf{J}}=\left(\begin{array}{cc}-1106 & 185 \\ 19 & -879\end{array}\right)$. The backlash deadbands have also been automatically estimated in-situ (see fig. 20). The results of the automatic compensation are presented in fig. 21. Since the main objective is the stabilization of the target in the image, we are mainly interested in the maximum excursion of the target. The amplitude of the disturbance before compensation is 218 pixels on the $\mathrm{X}$ image axis and 52.7 pixels on the $\mathrm{Y}$ image axis. The maximum residual errors after convergence are 13.9 pixels on the $\mathrm{X}$ image axis and 10.9 pixels on the $\mathrm{Y}$ image axis. The effect of the disturbance in the image is higly reduced: more than $90 \%$ on $\mathrm{X}$ and almost $80 \%$ on $Y$.

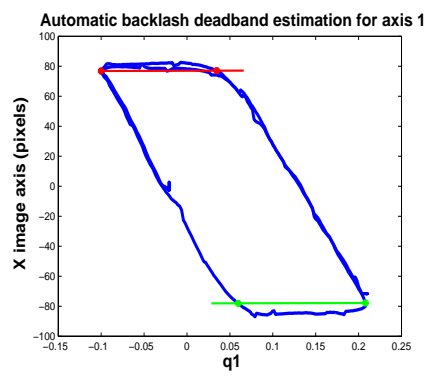

(a)

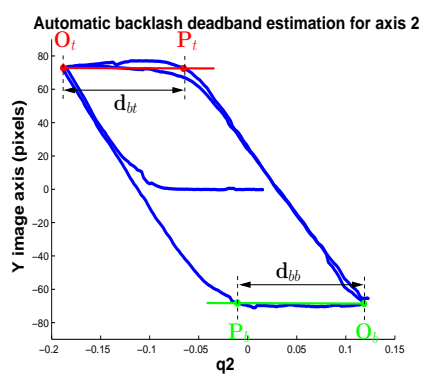

(b)
Fig. 20. Deadband identification during a test bed experiment. For axis 1, the identified values are $d_{b t}=0.1347 \mathrm{rad}, d_{b b}=0.1482 \mathrm{rad}$. For axis 2, the values are $d_{b t}=0.1226 \mathrm{rad}$ and $d_{b b}=0.1290 \mathrm{rad}$.
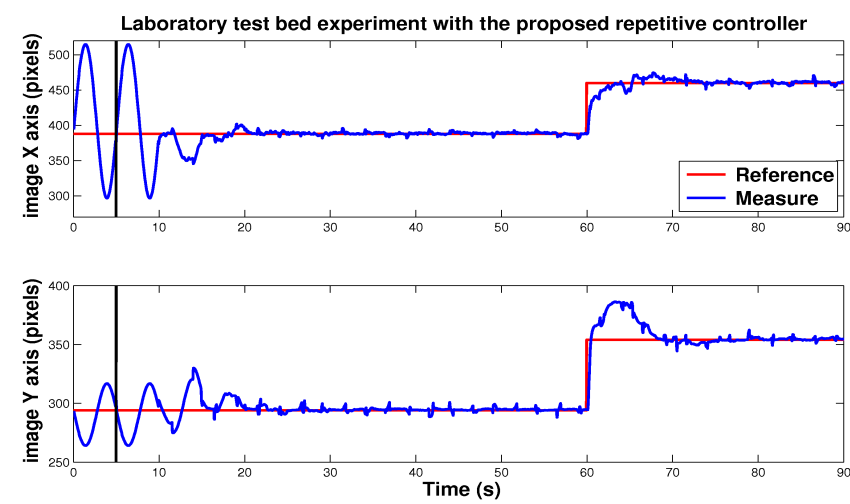

Fig. 21. Laboratory test bed experiment with the repetitive controller. The controller is activated at $t=5 \mathrm{~s}$. A reference step is applied at time $t=60 \mathrm{~s}$.

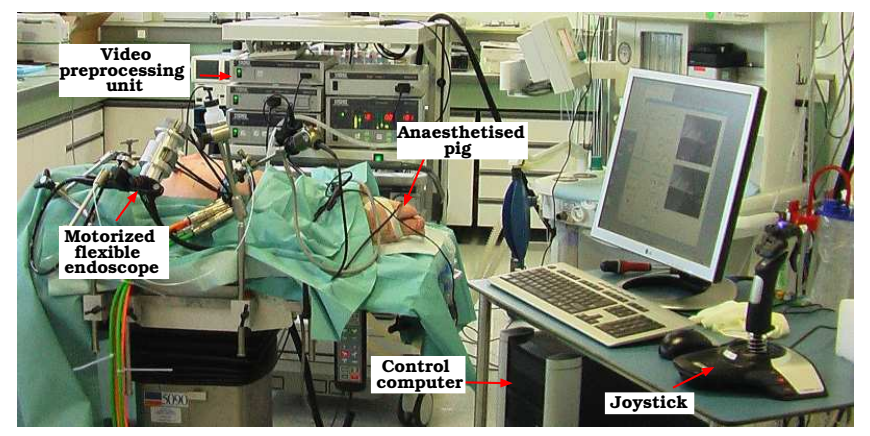

Fig. 22. In vivo experiment setup.

\section{E. In Vivo experiments}

Several in vivo experiments have been performed at the IRCAD $^{4}$ on anaesthetized pigs placed under artificial ventilation $^{5}$. The motorized endoscope was inserted into the abdominal cavity by passing through a trocart (see fig. 22). The presented experiments have been carried out on the liver which is subject to large breathing motions. The image target is a small burn spot onto the organ. Note that in this experiment, since the endoscope is constrained in the trocart, the breathing motion of period $4 s$ acts not only on the moving target, but also on the flexible shaft of the endoscope. In the given configuration, the jacobian matrix has been estimated to $\hat{\mathbf{J}}=\left(\begin{array}{cc}-1540 & 151 \\ -242 & -1456\end{array}\right)$. For the deadband, the identified values are $d_{b t}=0.1272 \mathrm{rad}, d_{b b}=0.1341 \mathrm{rad}$. for axis 1 and $d_{b t}=0.1032 \mathrm{rad}$ and $d_{b b}=0.1707 \mathrm{rad}$. for axis 2 Results of the visual servoing are presented on figure 23 . The amplitude of the disturbance before compensation is 179.6 pixels on the $\mathrm{X}$ image axis and 93.6 pixels on the $\mathrm{Y}$ image axis. The amplitude of the residual disturbance after convergence is 21.2 pixels on the $\mathrm{X}$ image axis and 16.9 pixels on the $\mathrm{Y}$ image axis, i.e. reduction of $88 \%$ and $82 \%$. The effect of the repetitive controller is very close to the behaviour observed on the laboratory testbed despite the high frequencies harmonics contained in the breathing disturbance signal (see fig. 14). The

\footnotetext{
${ }^{4}$ Institut de Recherche sur les Cancers de l'Appareil Digestif, Strasbourg, France

${ }^{5}$ See videos at http://eavr.u-strasbg.fr//lott/tro09.avi
} 
small residual error is due to the $Q$ filter which is necessary to ensure stability of the system but which decreases the capability of rejecting high frequencies harmonics due to the disturbance and the backlash. The behaviour during reference change is also very good, since the error is brought back to its steady state level in only two periods of the disturbance.
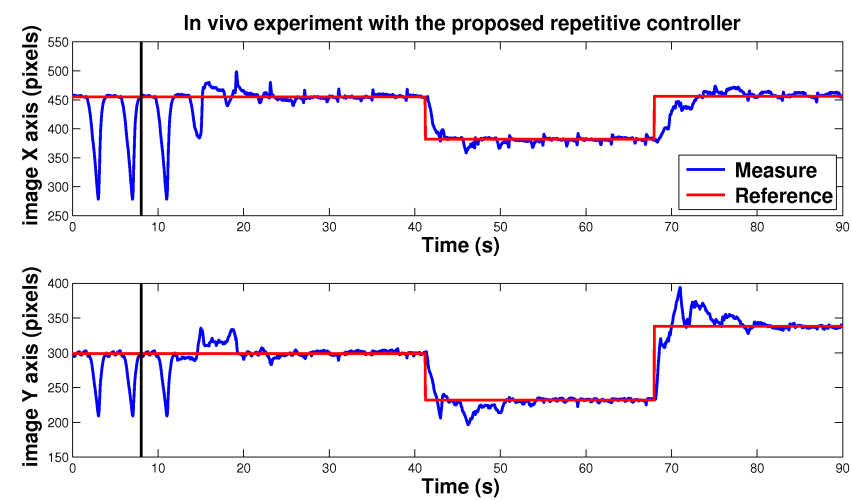

Fig. 23. In vivo experiment with the repetitive controller. The controller has been activated at $t=8 \mathrm{~s}$. Two reference changes are applied at $t=42 \mathrm{~s}$ and $t=68 s$.

\section{CONCLUSION}

We have shown in this paper that it is possible to control a flexible endoscope for breathing compensation using only the embedded vision system of the endoscope. The system is based on repetitive control and $2 \mathrm{D}$ visual servoing which requires no camera calibration. The theoretical and practical behaviour of the endoscopic system has been analyzed and an automatic method for estimating the linear and nonlinear components of the system has been proposed. The presented method for breathing compensation can hence be used without any expert tuning of the controller. In vivo experiments have been carried out on a pig model and they exhibit very promising results. The repetitive control algorithm has satisfying behaviour according to the surgeons who have assessed the system at the IRCAD in Strasbourg. The proposed approach can be used for stabilizing the endoscopic view in transluminal operations and hence decrease the number of surgeons manipulating the system. There are also other possible applications in classical gastroscopy for example for stabilizing the endoscopic view during tumor resection.

\section{ACKNOWLEDGMENT}

This work has been supported by a grant from the Alsace Regional Council.

\section{REFERENCES}

[1] O. Bebek and M.C. Cavusoglu. Intelligent control algorithms for robotic-assisted beating heart surgery. IEEE Transactions on Robotics, 23(3):468-480, 2007.

[2] S. Benhimane and E. Malis. Homography-based 2d visual tracking and servoing. The International Journal of Robotics Research, 26(7):661676, 2007.

[3] J.Y. Bouguet. http://www.vision.caltech.edu/bouguetj/calib_doc. Website presenting the Camera calibration toolbox for matlab.
[4] F. Chaumette and S. Hutchinson. Visual servo control, part i: Basic approaches. IEEE Robotics and Automation Magazine, 13(4):82-90, 2006.

[5] A. I. Comport, E. Marchand, M. Pressigout, and F. Chaumette. Real-time markerless tracking for augmented reality: the virtual visual servoing framework. IEEE Trans. on Visualization and Computer Graphics, 12(4):615-628, 2006

[6] D. F. Dementhon and L. S. Davis. Model-based object pose in 25 lines of code. International Journal of Computer Vision, 15:123-141, 1995.

[7] B. A. Francis and W. M. Wohnam. The internal model principle for linear multivariable regulators. Appl. Math and Opt., 2:170-194, 1975.

[8] J. Gangloff, R. Ginhoux, M. de Mathelin, L. Soler, and J. Marescaux. Model predictive control for compensation of cyclic organ motions in teleoperated laparoscopic surgery. IEEE Transactions on Control Systems Technology, 14(2):235-246, 2006.

[9] B. A. Jones and I. D. Walker. Kinematics for multisection continuum robots. IEEE Transactions on Robotics, 22(1):43-55, 2006.

[10] C. W. Ko and A. N. Kalloo. Per-oral transgastric abdominal surgery. Chinese Journal of Digestive Diseases, 7(2):67-70, 2006.

[11] J. Marescaux, B. Dallemagne, and S. Perretta et al. Surgery without scars: Report of transluminal cholecystectomy in a human being. Archives of Surgery, 142(9):823-826, 2007.

[12] L. Ott, Ph. Zanne, Fl. Nageotte, M. de Mathelin, and J. Gangloff. Physiological motion rejection in flexible endoscopy using visual servoing. IEEE International Conference on Robotics and Automation, 2008.

[13] B. Panomruttanarug and R. W. Longman. Repetitive controller design using optimization in the frequency domain. AIAA/AAS Astrodynamics Specialist Conference and Exhibit, 2004.

[14] P. O. Park, M. Bergström, K. Kikeda, A. Fritscher-Ravens, and P. Swain. Experimental studies of transgastric gallbladder surgery : cholecystectomy and cholecystogastric anastomosis. Gastrointestinal Endoscopy, 61(4):601-606, 2005.

[15] C.N. Riviere, J. Gangloff, and M. de Mathelin. Robotic compensation of biological motion to enhance surgical accuracy. Proceedings of the IEEE, 94(9):1705-1716, 2006.

[16] G. Tao and F. L. Lewis. Adaptive control of nonsmooth dynamic systems. Springer, 2001.

[17] M. Tomizuka, T. C. Tsao, and K. Chew. Analysis and synthesis of discrete-time repetitive controllers. ASME J. of Dynamic Systems, Measurement, and Control, 111:353-358, 1989.

[18] Z. Zhang. Flexible camera calibration by viewing a plane from unknown orientations. International Conference on Computer Vision, 26(7):666673, 1999.

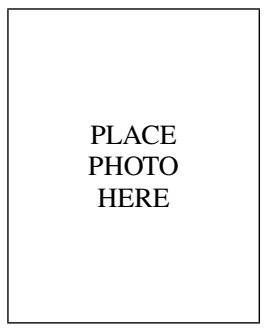

Laurent Ott Biography text here.

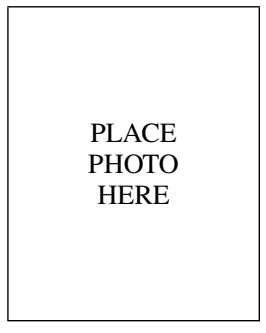


Philippe Zanne Biography text here.

PLACE

PHOTO

HERE

\begin{tabular}{|c|}
\hline \\
\\
PLACE \\
PHOTO \\
HERE \\
\end{tabular}

Michel de Mathelin Biography text here. 